\title{
Las dimensiones del material en piragüismo de aguas tranquilas Equipment dimensions in flatwater canoeing
}

\author{
Fernando Alacid Cárceles* \\ *Universidad de Murcia (España)
}

Resumen: El objetivo de artículo fue describir las diferentes formas de realizar la optimización de las dimensiones del material de competición utilizada en piragüismo de aguas tranquilas. En este trabajo se exponen los métodos tradicionales utilizados para determinar las dimensiones de la pala, así como las relaciones entre las características antropométricas y la longitud de la pala, la distancia entre ambas manos al agarrar la pala y la distancia entre el punto más bajo del asiento y el reposapiés.

Palabras clave: optimización del material, piragüismo, canoa, kayak.

Abstract: The objective of this paper was to describe the different ways to set-up the dimensions of the equipment used in flatwater canoeing. This article provides the traditional methods used in order to set up the paddle dimensions, and the relationships between anthropometric dimensions and the paddle length, the distance between hand grips, and the horizontal distance between the lowest point of the seat and the foot-bar.

Key words: equipment set-up, canoeing, canoe, kayak.

\section{Introducción.}

La optimización del material es algo fundamental en el deporte de competición para la obtención del máximo rendimiento posible, así como para la comodidad del deportista y la prevención de lesiones. La evaluación de los recursos materiales en el deporte se ha realizado tradicionalmente en actividades donde se utilizaba un implemento que era considerado como una pieza clave para el éxito deportivo, como por ejemplo en golf, hockey o salto con pértiga, existiendo además importantes trabajos en remo en los que se ha buscado la relación entre la configuración del material y las medidas antropométricas, la capacidad física, las variables cinemáticas de la remada y el rendimiento (Barrett \& Manning, 2004; Caplan \& Gardner, 2005).

En piragüismo de aguas tranquilas la embarcación se encuentra restringida en gran parte de sus medidas, mientras que la pala puede tener cualquier tamaño o forma, por lo que la optimización de sus dimensiones es clave para obtener el mayor rendimiento posible. Por ello, el objetivo de este trabajo es describir las diferentes aportaciones existentes en la literatura sobre la optimización del material de competición en piragüismo de aguas tranquilas y su relación con las dimensiones antropométricas de los palistas.

\section{Aproximación tradicional a las dimensiones la pala en} piragüismo de aguas tranquilas.

En piragüismo, tradicionalmente se han empleado las dimensiones corporales para la determinación de la longitud de la pala, ya Toro (1986), indicó la no existencia de fórmulas apropiadas para obtener la longitud de la pala ideal, aún así, propuso como la forma más utilizada para hallar la longitud idónea de la pala de kayak aquella en la que el palista es capaz de agarrar de uno de sus extremos si la pala se encuentra colocada en una posición vertical junto al palista, este método es el más utilizado en la actualidad, de hecho Szanto \& Henderson (2004) lo recomiendan como un buen punto de partida para elegir la longitud total de la pala, uniendo a sus consejos, la no utilización de otras medidas como la talla sentado, la anchura de hombros, la longitud del brazo o la fuerza del sujeto para su determinación. Sin embargo para Sánchez \& Magaz (1993), la determinación exacta de esta dimensión parte de un proceso de análisis del paleo basado en los siguientes aspectos:

- Respetar unángulo correcto de entrada de la pala durante el ataque. - La trayectoria del brazo de empuje debe ser paralela al agua, ya que una pala demasiado larga produce que se eleve excesivamente la mano por encima de la cabeza.

Fecha recepción: 15-03-08 - Fecha envío revisores: 17-03-08 - Fecha de aceptación: 29-05-08 Correspondencia: Fernando Alacid Cárceles

$\mathrm{C} /$ Argentina, $\mathrm{s} / \mathrm{n}$.

30720, Santiago de la Ribera (Murcia)

E-mail: fernando.alacid@um.es
- Una pala demasiado larga impide obtener la máxima frecuencia de paleo.

- Las sensaciones del palistas en cuanto a poder aplicar la máxima fuerza en cada palada y la posibilidad de incrementar la frecuencia de paleo durante los finales de las pruebas o en situaciones de fatiga.

En la modalidad de canoa canadiense, la norma más habitual para determinar la longitud de la pala se obtiene tras colocar la misma delante del palista en posición vertical, ésta debe llegar aproximadamente a la altura de los ojos o de las cejas, por lo que la medida antropométrica más utilizada para obtener esta medida es la altura del canoista (Sánchez \& Magaz, 1993; Szanto \& Henderson, 2004; Toro, 1986). Toro (1986), además, propuso otros métodos colocando al palista en su posición habitual de paleo y con la pala vertical apoyada en el suelo, el brazo de gobierno debía de ser capaz de llegar a coger la empuñadura de la pala.

Para obtener el agarre de la pala de kayak, tradicionalmente se ha colocado la pala sobre la cabeza, y manteniendo la misma distancia entre las manos y cada una de las hojas, ambos codos deben estar flexionados $90^{\circ}$ (Szanto \& Henderson, 2004; Toro, 1986), método ampliamente difundido en piragüismo ya que es utilizado en otras modalidades como las aguas bravas (Ferrero, 2006). Para Sánchez \& Magaz (1993), la obtención del agarre de la pala de canoa es similar a la utilizada en kayak, con laúnica diferencia de que el codo de gobierno se encuentra flexionado $90^{\circ}$ y el de tracción $80^{\circ}$, por lo que el agarre en canoa es ligeramente más cerrado que en kayak.

Para la determinación de la longitud y anchura de la hoja, el palista en función de su edad, nivel de aprendizaje, fuerza y estilo paleando, debe probar diferentes dimensiones de la hoja, para determinar, junto con su entrenador, la que mejor se adapta a su forma de palear (Szanto $\&$ Henderson, 2004). Toro (1986), incluso aportó datos sobre las dimensiones de las palas de kayak y de canoa en función del tipo de embarcación en el que se competía (tabla 1), sugiriendo el uso de palas y hojas mayores en las embarcaciones colectivas en la modalidad de kayak, y longitudes de la pala y hoja menores y anchura de la hoja superiores en las embarcaciones colectivas de canoa. Es necesario tener en cuenta que el modelo de pala al que hace referencia Toro (1986) ha cambiado mucho respecto a las utilizadas en la actualidad, especialmente en kayak, donde incluso se basan un principio físico diferente para propulsar la embarcación.

De cualquier forma, el proceso de elección de las dimensiones de la hoja suele estar guiado fundamentalmente por las recomendaciones de los fabricantes de palas, puesto que desarrollan modelos orientados a las diversas categorías existentes.

Como se ha venido exponiendo hasta el momento, la configuración de la pala no se encuentra sometida a ninguna regulación especial en ambas modalidades, excepto que no pueden existir estructuras utilizadas como ejes de palanca entre la pala y la embarcación (ICF, 2007; RFEP, 2007). Sin embargo, las embarcaciones se encuentran restringidas en cuanto a sus características, especialmente en longitud y forma, por lo que las posibilidades de adaptación de las mismas a las dimensiones del 


\begin{tabular}{cccccccc}
\hline \multicolumn{4}{c}{ Tabla 1. Orientaciones sobre las dimensiones de la pala. Tomada de Toro (1986) } \\
\hline \multirow{2}{*}{ Dimensiones } & \multicolumn{2}{c}{ Hombre Kayak } & \multicolumn{3}{c}{ Mujer Kayak } & \multicolumn{3}{c}{ Hombre Canoa } \\
\cline { 2 - 8 } & K-1 & K-2 /-4 & K-1 & K-2/-4 & C-1 & C-2 & C-4/-7 \\
\cline { 2 - 8 } Longitud de la pala $(\mathrm{cm})$ & $217-224$ & $220-226$ & $215-222$ & $218-224$ & $160-185$ & $160-185$ & $155-175$ \\
Largo de la hoja $(\mathrm{cm})$ & $47-50$ & $46-52$ & $45-48$ & $45-48$ & $60-80$ & $55-70$ & $50-65$ \\
Ancho de la hoja (cm) & $17-20$ & $18-22$ & $15-18$ & $16-19$ & $17-20$ & $18-22$ & $19-23$ \\
\hline
\end{tabular}

口 $\mathrm{E} 1=(0.438 \cdot$ Talla $)+(0.731 \cdot$ Longitud de la Pierna $)-0.224 \mathrm{~m}$

口 $\mathrm{E} 2=(0.603 \cdot$ Talla $)-0.160 \mathrm{~m}$

口 $\quad$ E3 $=(0.454 \cdot$ Talla $)+(0.295 \cdot$ Longitud de la Pierna $)-(0.375$. Longitud del pie $)+(0.403 \cdot$ Longitud del Muslo $)-0.11882 \mathrm{~m}$

De un modo similar, para el agarre de la pala la ecuación predictora

palista suelen ser realizadas en las fábricas, desarrollando diferentes modelos de embarcaciones según el peso del palista o la anchura de las caderas, en el caso del kayak. Por ello, una de las pocas dimensiones a configurar en esta modalidad es la distancia entre el asientoy el reposapiés. Medida condicionada, principalmente, por la técnica de paleo, ya que en una posición inicial, las piernas deben encontrarse ligeramente flexionadas $\left(110-120^{\circ}\right)$, permitiendo el trabajo de pedaleo sin llegar en ningún momento a la extensión completa (Sánchez \& Magaz, 1993), por ello, el principal criterio utilizado para la obtención de esta distancia es la comodidad en el movimiento de propulsión de las piernas.

\section{Optimización del material de competición en palistas} olímpicos.

La primera aproximación científica a la optimización del material fue realizada por Ong et al. (2005; 2006), donde en el primero de sus trabajos (Ong et al., 2005) analizaron las dimensiones de la pala y la configuración del asiento en los kayakistas que participaron en los JJ.OO. de Sydney (2000) con el fin de establecer las posibles diferencias entre las aguas tranquilas y el slalom, y entre los mejores palistas y el resto de participantes, así como buscar las posibles correlaciones con diferentes medidas antropométricas. Mientras que en el segundo utilizaron las correlaciones determinadas previamente para realizar un análisis de técnica de tres palistas empleando diferentes configuraciones del material (Ong et al., 2006).

En la tabla 2, se exponen las diferentes dimensiones de la pala, la distancia entre el punto más bajo del asiento y el reposapiés, la distancia del asiento a la parte más baja del casco de la embarcación y a la parte superior de la bañera y la talla de los palistas, encontradas por Ong et al. (2005) en los kayakistas olímpicos de aguas tranquilas y slalom.

Como se puede observar, las diferencias entre aguas tranquilas y slalom se encontraron en la distancia del asiento al fondo del casco de la embarcación y en las dimensiones de la pala, ya que los palistas de slalom ubican el centro de gravedad mucho más bajo que los de aguas tranquilas, utilizan palas unos $15 \mathrm{~cm}$ más cortas, y las hojas poseen una forma diferente al no poseer una curvatura tan acentuada como las de aguas tranquilas y ser más cortas y anchas que las de aguas tranquilas. Por otro lado, las diferencias entre la configuración del material entre los 15 mejores hombres kayakistas y el restoúnicamente fueron significativas en la distancia del asiento al borde superior de la bañera, con valores más altos en los mejores palistas.

En este mismo estudio, desarrollaron ecuaciones predictoras de la distancias entre el punto más bajo del asiento y el reposapiés y del agarre de la pala(Ong et al., 2005). Para el primero de los casos (asiento - reposapiés) aportaron dos ecuaciones, una para todos los hombres kayakistas de aguas tranquilas y aguas bravas (E1) que explicaba un $48 \%$ de la varianza y otra a partir de los datos de los hombres kayakistas de aguas tranquilas (E2), con una predicción de la varianza cercana al $60 \%$. Con un valor de predicción similar, Ong et al. (2006), aportaron una tercera ecuación en la que utilizaban además de la talla, las longitudes del muslo, la pierna y el pie (E3).

\begin{tabular}{|c|c|c|c|c|}
\hline \multicolumn{5}{|c|}{$\begin{array}{l}\text { Tabla 2. Dimensiones del material y talla en kayakistas participantes en los JJ.OO. de Sydney (2000). } \\
\text { Tomada de Ong et al. (2005) }\end{array}$} \\
\hline \multirow[b]{2}{*}{ Variables } & \multicolumn{2}{|c|}{ Aguas Tranquilas } & \multicolumn{2}{|c|}{ Slalom } \\
\hline & $\begin{array}{c}\text { Hombres } \\
(\mathrm{n}=31)\end{array}$ & $\begin{array}{l}\text { Mujeres } \\
(\mathrm{n}=11)\end{array}$ & $\begin{array}{c}\text { Hombres } \\
(\mathrm{n}=12)\end{array}$ & $\begin{array}{l}\text { Mujeres } \\
(\mathrm{n}=12)\end{array}$ \\
\hline Talla $(\mathrm{cm})$ & $184.5 \pm 6.0$ & $168.6 \pm 6.0$ & $177.1 \pm 6.6$ & $167.7 \pm 5.2$ \\
\hline Longitud de la pala $(\mathrm{cm})$ & $220.2 \pm 1.7$ & $215.3 \pm 1.7$ & $203.0 \pm 1.6$ & $199.5 \pm 1.8$ \\
\hline Largo de la hoja (cm) & $51.5 \pm 1.1$ & $50.4 \pm 1.7$ & $47.0 \pm 1.2$ & $47.0 \pm 0.9$ \\
\hline Ancho de la hoja (cm) & $17.0 \pm 0.4$ & $16.5 \pm 0.6$ & $20.0 \pm 0.6$ & $19.3 \pm 0.4$ \\
\hline Agarre de la pala $(\mathrm{cm})$ & $72.8 \pm 3.7$ & $67.9 \pm 4.7$ & $70.3 \pm 6.3$ & $66.3 \pm 6.0$ \\
\hline Distancia asiento-reposapiés $(\mathrm{cm})$ & $94.9 \pm 6.1$ & $87.2 \pm 6.0$ & $88.1 \pm 2.8$ & $84.7 \pm 3.7$ \\
\hline Distancia asiento-casco $(\mathrm{cm})$ & $8.2 \pm 1.2$ & $7.6 \pm 1.3$ & $0.8 \pm 0.6$ & $0.6 \pm 0.5$ \\
\hline Distancia asiento-bañera $(\mathrm{cm})$ & $20.8 \pm 1.4$ & $20.5 \pm 1.3$ & $21.7 \pm 1.2$ & $20.9 \pm 0.7$ \\
\hline
\end{tabular}
desarrollada para todos los hombres kayakistas de aguas tranquilas y aguas bravas (E4) explicaba un $40 \%$ de la varianza, mientras que la obtenida únicamente para los palistas de aguas tranquilas (E5) lo hacía con un mayor nivel de predicción $\left(r^{2}=0.541\right)$.

口 $\mathrm{E} 4=(0.422 \cdot$ Talla $)+(0.559 \cdot$ Diámetro transverso del tórax $)-$ $(0.175 \cdot$ Envergadura $)+0.102 \mathrm{~m}$

$\square \quad \mathrm{E} 5=(0.376 \cdot$ Talla $)+0.0356 \mathrm{~m}$

Unavezexaminada el poder explicativo de cada una de las ecuaciones, y teniendo en cuenta la temática de este trabajo, las ecuaciones E2 y E5 parecen las más adecuadas para la predicción de las medidas estudiadas en hombres palistas de aguas tranquilas, además de necesitarúnicamente la talla para obtener la distancia requerida. En este mismo sentido Ong $e t$ al. (2006) aportaron nuevas correspondencias entre las variables relacionadas con la configuración del material y las antropométricas expresando unas como el porcentaje de otras, de esta forma:

口 Agarre $=32.6 \pm 1.8 \%$ de la longitud total de la pala; $=61.4 \pm 3.0 \%$ de la longitud de la pértiga; $=170.2 \pm 11.0 \%$ del diámetro biacromial

- Longitud de la pala $=121.4 \pm 4.9 \%$ de la talla

口 Longitud de la pala $=118.3 \pm 6.0 \%$ de la envergadura

Distancia del asiento al reposapiés $=51.4 \pm 2.5 \%$ de la talla

口 Distancia del asiento al reposapiés $=98.2 \pm 4.8 \%$ de la longitud de la extremidad inferior

En el trabajo de Ong et al.(2006), realizaron un análisis de la técnica de paleo bajo tres situaciones diferentes de configuración del material, una con la preferida de los palistas, otra con la obtenida aplicando las ecuaciones E2 y E5 descritas anteriormente y una tercera sumando una desviación típica a los valores obtenidos por las ecuaciones predictoras.

La muestra estuvo compuesta por tres sujetos que realizaron un test de $100 \mathrm{~m}$ a una velocidad similar a una prueba de $500 \mathrm{~m}$. Al tratarse de un numero de palistas tan escaso, los resultados obtenidos han de ser interpretados con precaución, pero como norma general, la configuración del material preferida por los palistas obtuvo valores superiores de velocidad y de frecuencia de ciclo respecto al resto de configuraciones, mientras que la longitud de ciclo fue superior en las configuraciones obtenidas mediante las ecuaciones predictoras. Estos cambios, especialmente el producido en la velocidad, fueron originados por las alteraciones de la mecánica de paleo al cambiar el agarre de la pala y la distancia del asiento al reposapiés. Tampoco se encontraron mejoras en el tiempo de impulsión al usar configuraciones diferentes a la preferida por los palistas.

Como recomendación final, los autores consideran que el uso de las ecuaciones predictoras del agarre de la pala y la distancia del asiento al reposapiés pueden aplicarse en momentos fuera de la temporada competitiva y realizar un análisis pormenorizado de los efectos conseguidos para asimilar o desechar los cambios realizados.

\section{Referencias bibliográficas.}

Barrett, R. S., \& Manning, J. M. (2004). Relationships between rigging set-up, anthropometry, physical capacity, rowing kinematics and rowing performance. Sports Biomech, 3(2), 221-235.

Caplan, N., \& Gardner, T. (2005). The influence of stretcher height on the mechanical effectiveness of rowing. J Appl Biomech, 21, 286-296.

Ferrero, F. (2006). Coaching handbook. Gwynedd: Pesda Press.

ICF. (2007). Flatwater racing competition rules. Consultado el 14-06-2007, disponible en www.canoeicf.com

Ong, K., Elliott, B., Ackland, T., \& Lyttle, A. (2006). Performance tolerance and boat setup in elite sprint kayaking. Sports Biomech, 5(1), 77-94.

Ong, K. B., Ackland, T. R., Hume, P.A., Ridge, B., Broad, E., \& Kerr, D.A. (2005). Equipment set-up among Olympic sprint and slalom kayak paddlers. Sports Biomech, 4(1), 4758.

RFEP. (2007). Reglamento General y Técnico de Competiciones. Consultado el 10-052007, disponible en www.rfep.es

Sánchez, J. L., \& Magaz, S. (1993). La Técnica. En J. L. Sánchez(Ed.), Piragüismo (I) (pp. 101-386). Madrid: COE.

Szanto, C., \& Henderson, D. (2004). Flatwater racing. Level 1. Beginning coach. Madrid: International Canoe Federation.

Toro, A. (1986). Canoeing: an olympic sport. San Francisco: Olympian Graphics. 